\title{
STUDY OF THE ENERGY PARAMETERS AND POSSIBILITIES FOR MODERNIZATION OF ELECTRIC VEHICLES FOR AC POWER
}

The main problem with thyristor locomotives operated in the country is the low value of the power factor. The paper presents results of a survey of the quality of power consumption in this type of locomotives. Based on the experimental data analyzed, we have determined the main parameters characterizing the traction power consumption. Indicated are some general guidelines for optimization.

Keywords: Electric locomotives with thyristor rectifier, Power factor, Reactive Power Compensator.

\section{Introduction}

The main requirement for AC-driven electric vehicles (EV) such as locomotives and electric multiple units (EMU) in operation in the railway system is to have the capability of smooth regulation of traction and braking forces by means of suitable control gear (CG) allowing the vehicles to maintain their energy parameters (i.e. power coefficient $k_{M}$ ) at high levels across the entire operational range. The power coefficient at non-sinusoidal current and voltage is calculated using the following formula:

$$
\begin{aligned}
& k_{M}=\frac{P_{(1)}}{S}=\frac{U_{(1)} \cdot I_{(1)} \cdot \cos \varphi_{(1)}}{U_{e f f} \cdot I_{e f f}}, \text { with } U_{(1)} \approx U_{e f f}, \\
& k_{M}=\frac{U_{e f f} \cdot I_{(1)} \cdot \cos \varphi_{(1)}}{U_{e f f} \cdot I_{e f f}}=\nu_{1} \cdot \cos \varphi_{(1)},
\end{aligned}
$$

where:

$U_{(1)}, I_{(1)}, \cos \varphi_{(1)}$ and $P_{(1)}$, are respectively the effective values of voltage and current of the $1^{\text {st }}$ harmonic, the phase angle between them, and the active power carried by the main harmonic, $\mathrm{W}$; $U_{\text {eff }}, I_{\text {eff }}$ - the actual effective (True rms) voltage and current; $v_{I}$ - the current non-sinusoidality coefficient, $v_{I}=I_{(1)} / I_{\text {eff }}$; $S$ - the apparent power, VA.

A large percentage of the electric vehicles used in Bulgaria's railway system have diode and thyristor control gear (current rectifiers) and direct-current motors. Only the SIEMENS DESIRO Classic electric railcars by Siemens are equipped with four-quadrant power converters with IGBT, which allow for regulation of the power factor and propulsion by induction motors.

The main problem which all alternating current EVs with DC drives face, irrespective of their particular solution for regulating the mode of operation, are the deteriorated energy parameters. The combination of increased consumption of reactive power and the deformation of the propulsion current curve leads to a lower power coefficient. All of this is further compounded by the additional losses in the traction power supply system thereby decreasing its efficiency.

It is a well known fact that Bulgaria's rolling stock is worn out and technologically obsolete. It needs urgent renovation by replacing part of the EV fleet and upgrading certain vehicles with IGBT [1] converters. Of course, for a vehicle to be eligible for upgrade, it must have a sufficient residual operational life. This should be determined by a detailed preliminary assessment of the technical status of the available rolling stock.

For the EVs with diode converters used in the Bulgarian railway system (locomotives of the 44 and 46 series) increasing km can be achieved by remodeling their power circuitry to retrofit power inverters but leaving most of the propulsion installation intact. For the locomotives of the 44-001 and 46-200 series previously upgraded with thyristor rectifiers this approach would require for the rectifiers to be removed, which would not be economically justifiable. A better solution for them would be to outfit them with active compensators for reactive power connected to the secondary windings of the locomotive's propulsion transformer.

This paper is based on data from experimental measurements of the energy parameters of locomotives with thyristor rectifiers conducted in real-life operational environment. It proposes technical solutions for upgrading this type of EVs to decrease their consumption of reactive power and improve their power consumption profile.

\footnotetext{
* Georgy Pavlov

Transportation Power Supply and Power Equipment Department of the Todor Kableshkov University of Transport, Bulgaria, Sofia,

E-mail: g_pavlov61@abv.bg
} 


\section{Experimental study of the energy parameters of an upgraded, series 46-200 locomotive}

Since 1998 the KONČAR - ELEKTRIČNE LOKOMOTIVE d.d. factory in Zagreb, Croatia has upgraded 13 locomotives from the BDZ fleet. These include 2 locomotives from the 44 series and 11 from the 46 series (their codes have been amended as follows: 44-001 and 44-002, and Series 46-200). The upgrade consisted in retrofitting thyristor rectifiers with natural commutation and twozone voltage adjustment capability. In terms of energy performance, this solution is not optimal and leads to deterioration in the power consumption indicators.

There are a wide range of technical solutions for improving the energy parameters of EVs with thyristor current rectifiers (singlemode thyristors). However, only two of them seem to be favored in the industry. The first relies on using multi-zone adjustment (up to four adjustment zones) and yields relatively good results in terms of $k_{M}$. Increasing the number of the adjustment zones provides for better energy parameters (increased $k_{M}$ ) but makes the construction of the propulsion transformer increasingly more complicated and is therefore avoided. The second solution for increasing $k_{M}$ relies on the use of artificial commutation in the thyristor rectifiers. It offers a significant improvement of the EVs energy parameters [2]

To study and analyze the energy parameters of the upgraded EVs from the BDZ fleet, we conducted experimental measurements on trains drawn by 46-200 Series locomotives across the territory of pre-selected traction power substations and feeders. The measurements were performed using microprocessor measurement devices (MC 750 and UMG 604E grid analyzers). The devices performed real-time measurement of voltage and current. The data was then used to calculate the effective electric power (active, reac- tive, and apparent) and the power factor. A harmonic analysis of the propulsion current and voltage curves in the overhead lines is performed [3]. The measurements were taken with an integration period of 1 minute. All specified values were stored in the devices' onboard memory chips. The analyzers were connected to the traction power supply system via $800 / 5$ A current transformers and $35000 / 100 \mathrm{~V}$ voltage transformers.

We presented the results from measurements conducted in the area of Iliantsi Propulsion Power Substation on a train drawn by an upgraded, Series 46-200 locomotive with rated traction power of $5400 \mathrm{~kW}$. No other trains were operating in the area during the measurements. Based on the data gathered during the measurements, we were able to determine the main energy parameters for the studied type of locomotive.

Figure 1 shows the change in propulsion current $I_{e l}$, A of the locomotive between 12:00 PM and 12:50 PM. This time of day was selected because it featured all main movement patterns of the train such as acceleration according to a selected algorhythm, cruising at a predetermined speed, and dynamic resistor braking.

Figure 2 shows the changes in the active $(P, \mathrm{~W})$, reactive $(Q$, $\mathrm{VAr}$ ) and apparent ( $S, \mathrm{VA}$ ) power of the train. We witnessed a significant consumption of reactive power with inductive nature, which was similar in its value to the consumed active power. Within the time interval subject of our study the train had performed several run sequences for each of which the train engineer had chosen different algorhythms. During each of those, as we observed, the ratio between the reactive and active power changed and between 12:16 PM and 12:30 PM the reactive power had higher values than the active. From the charts in Figs. 2 and 3 we can conclude that to a large degree these values for the active and reactive power depend on the pattern of movement of the EV.

Iel, A

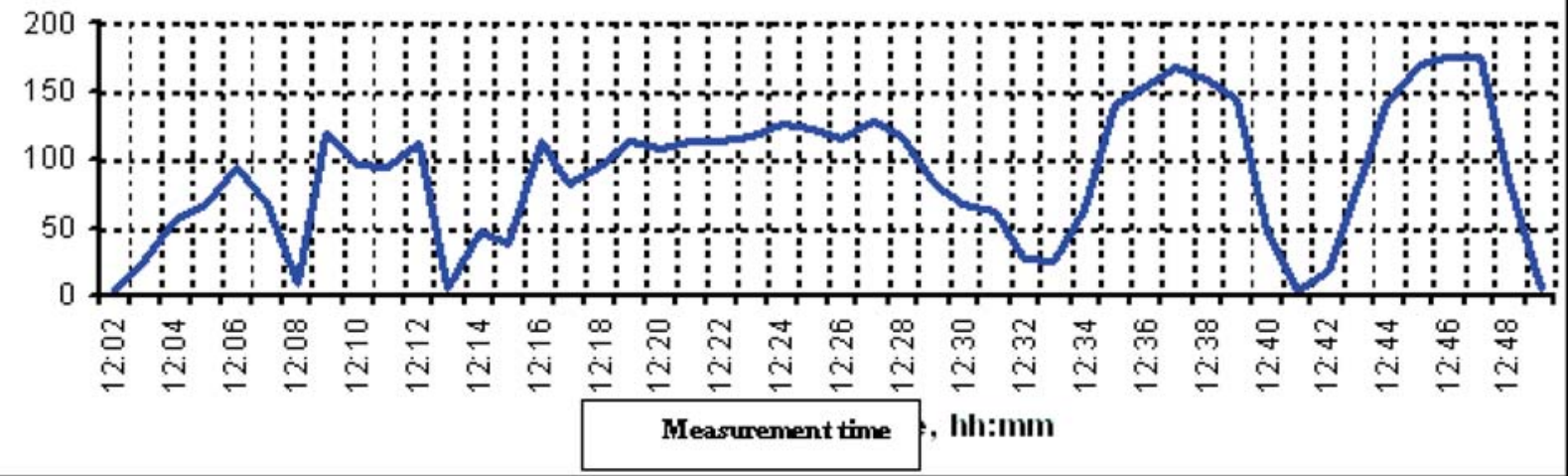

Fig. 1 Locomotive Propulsion Current Dynamics 


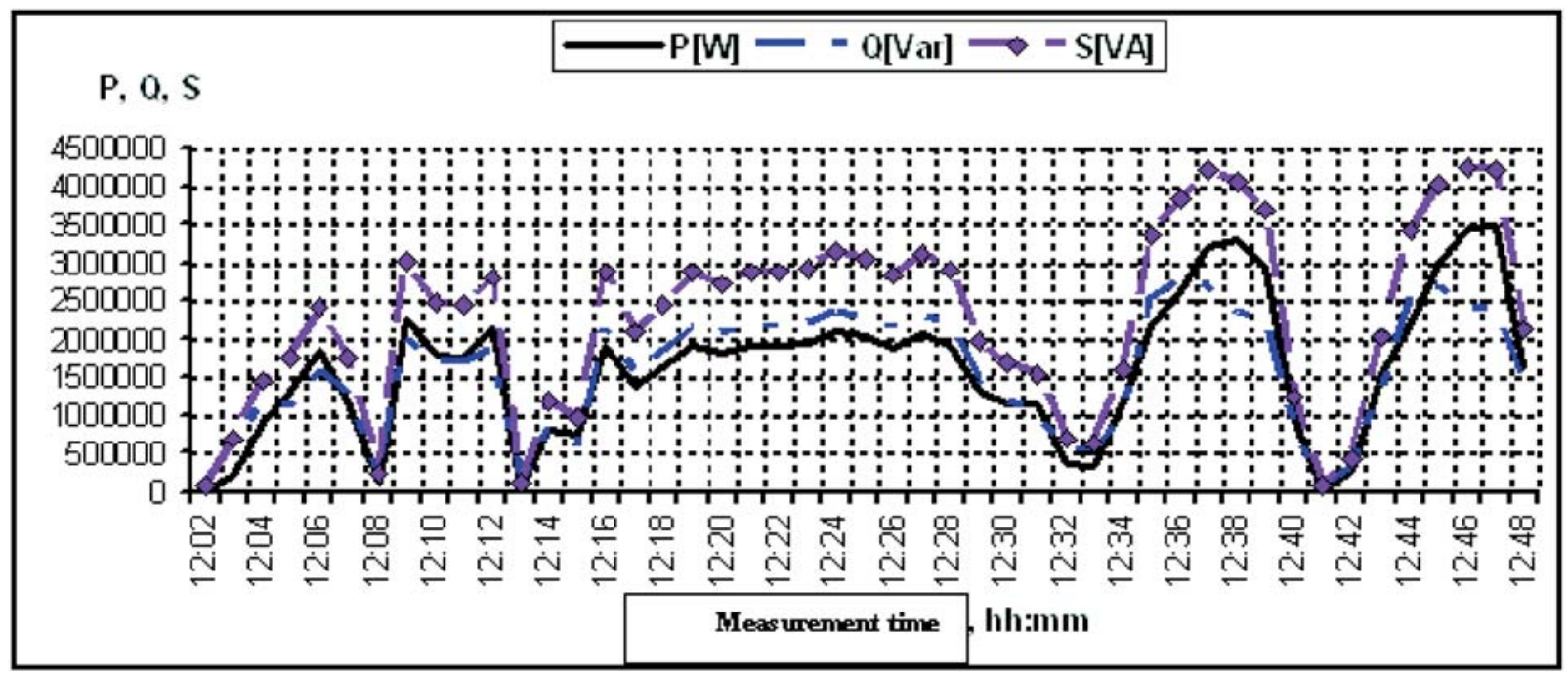

Fig. 2 Locomotive Active, Reactive, and Apparent Power Dynamics

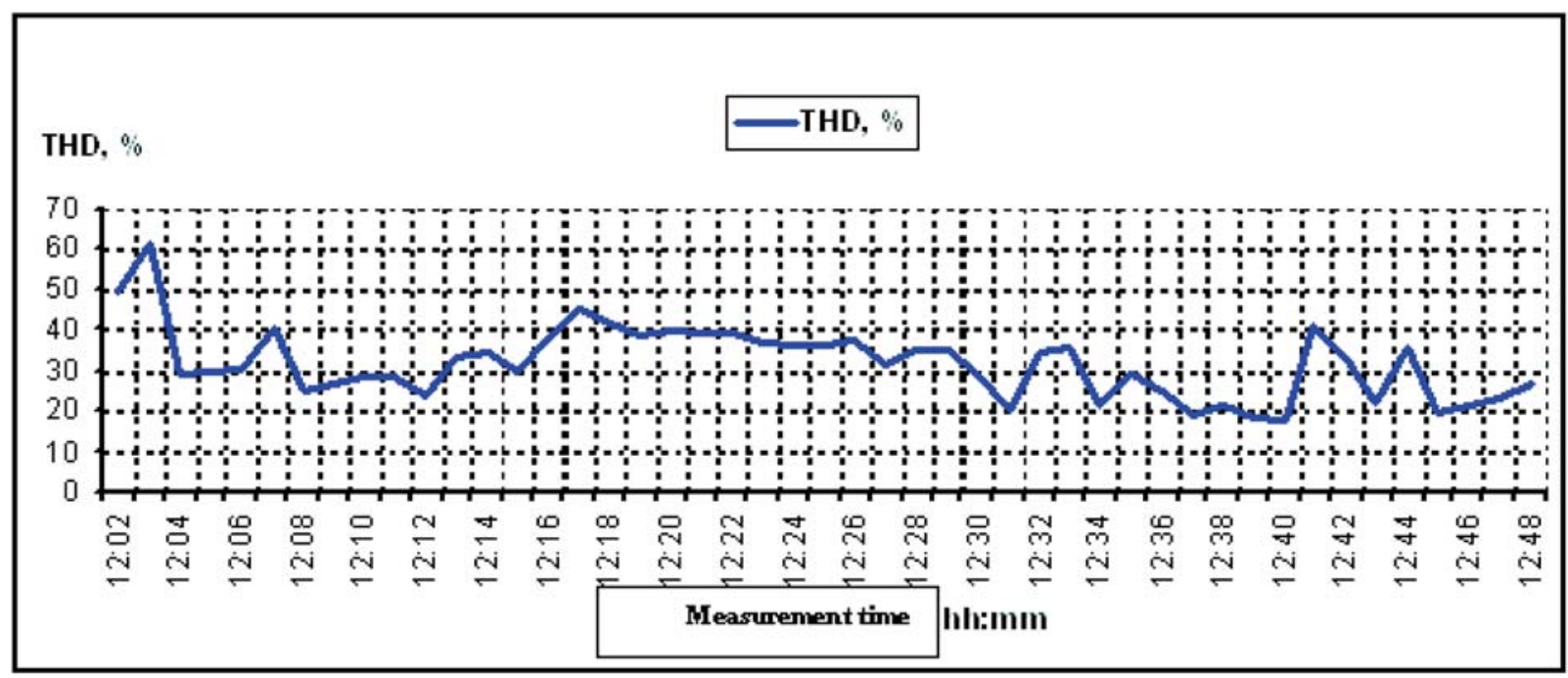

Fig. 3. Propulsion Current Harmonic Distortion Coefficient Dynamics

Figure 3 provides a graphical representation of the dynamics of the $T H D_{I}$, \% indicator (total harmonic distortion) of the propulsion current as measured at the $27.5 \mathrm{kV}$ terminal of the traction power supply substation. This indicator characterizes the distortion of the current curve and the presence of high-order harmonics as a percentage of the effective value of the current of the first harmonic $I_{(1)}$.

Over the specified time interval the value of THDI, \% varies widely (from $20 \%$ to $60 \%$ ) depending on the movement pattern of the train and the current value of the power. By using the calculation device [4] and formula (2) below, we managed to determine the change in the non-sinusolidality coefficient of the propulsion current $(\mathrm{vI})$, which itself varied between 0.858 and 0.98 .

$$
\begin{aligned}
& \nu_{I}=\frac{1}{\sqrt{1+\left(\frac{T H D_{I} \%}{100}\right)}}=\frac{1}{\sqrt{1+\left(\frac{\left.\sqrt{\sum_{i=2}^{\infty} I_{(1)}^{2}}\right)^{2}}{I(1)}\right)^{2}}}= \\
& =\frac{1}{\sqrt{\left(\frac{I_{e f f}^{2}}{I_{(1)}^{2}}\right)}}=\frac{I_{(1)}}{I_{e f f}}
\end{aligned}
$$

In addition to the above, we studied the changes in power factor $P F_{e l}$ and the results are presented in Fig. 4. Due to the low content of higher-order harmonics in the voltage supplied $\left(T H D_{U} \%<\right.$ $<10 \%$ ), the measured $P F_{e l}$ (in accordance with the method described in [4]) almost coincided with the power coefficient $k_{M}$, 


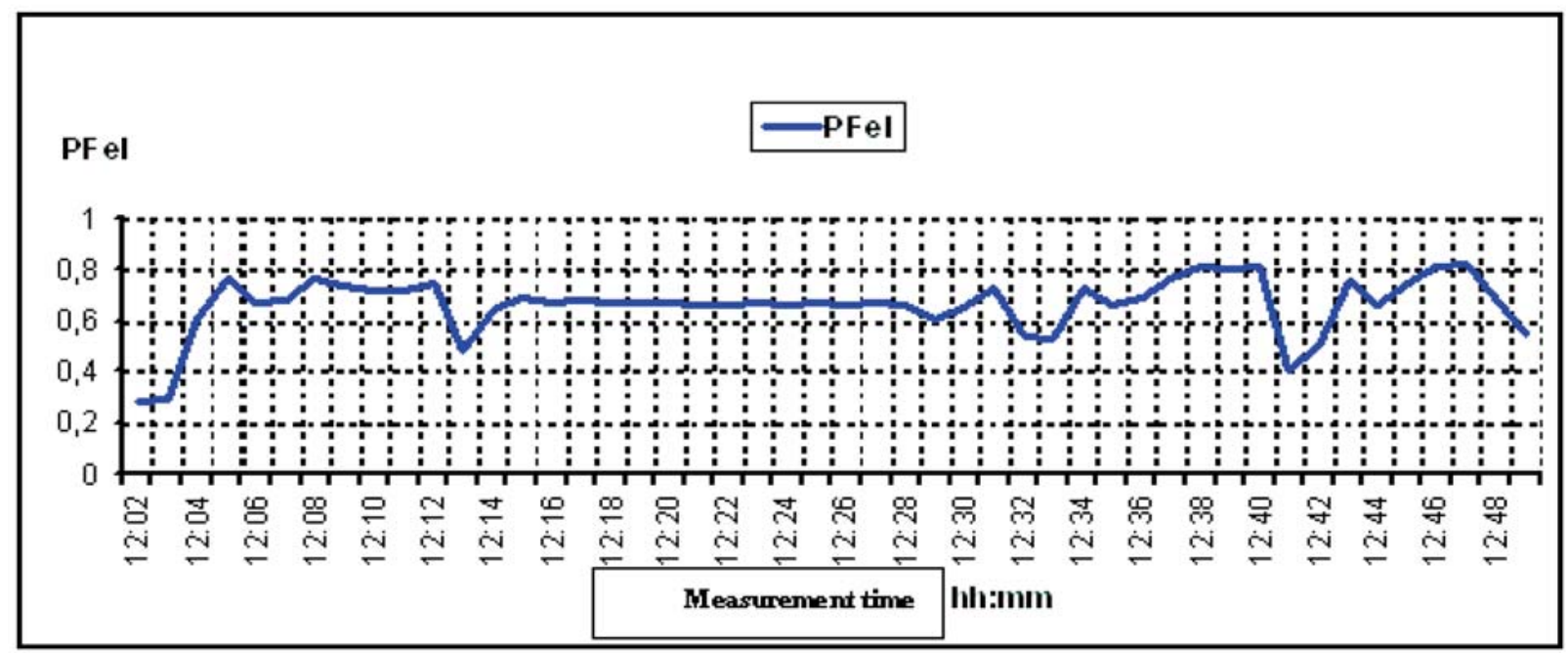

Fig. 4 Locomotive Power Factor Dynamics

i.e. $P F_{e l} \approx k_{M}$. The chart shows that for the most part of the measurement interval it remained under 0.8. Between 12:19 and 12:22, when the train had a relatively steady rate of power consumption, the average value of $P F_{e l}$ remained around 0.67. By a more detailed analysis of the measurement data we determined $\cos \varphi_{(1)}$ to be equal to 0.72 and the nonsinusoidality coefficient of the propulsion current $\left(v_{I}\right)$ to be equal to 0.93 . On the basis of these results we inferred that the low $k_{M}$ value is to a significant degree the result of the consumption of reactive power. We must also note that under this movement pattern, the locomotive's current power was about $36 \%$ of its rated power. During the intervals 12:37 PM to $12: 39 \mathrm{PM}$ and 12:45 PM to 12:47 PM when the locomotive was operating at over $60 \%$ of its rated power, these parameters had the following values: $\cos \varphi_{(1)} \approx 0.81 ; v_{I}, \approx 0.98$; and $P F_{e l}$, respectively $k_{M}$ was equal to $0.80-0.81$. This indicates that increasing the power leads to a significant improvement of the energy parameters of the EV. However, in a real-life operational environment this type of movement pattern is difficult to achieve.

During our studies we conducted numerous measurements on locomotives from the 44-001 and 46-200 Series. Different measurements on different trains under identical patterns of movement showed no significant differences of the power coefficients in terms of shape or form. The results gathered were completely consistent with those from similar measurements conducted within other studies [5].

Under the existing scheme, after the upgrade of the studied locomotives, the best way to improve their energy parameters is to retrofit them with active compensators for reactive power (ACRP)

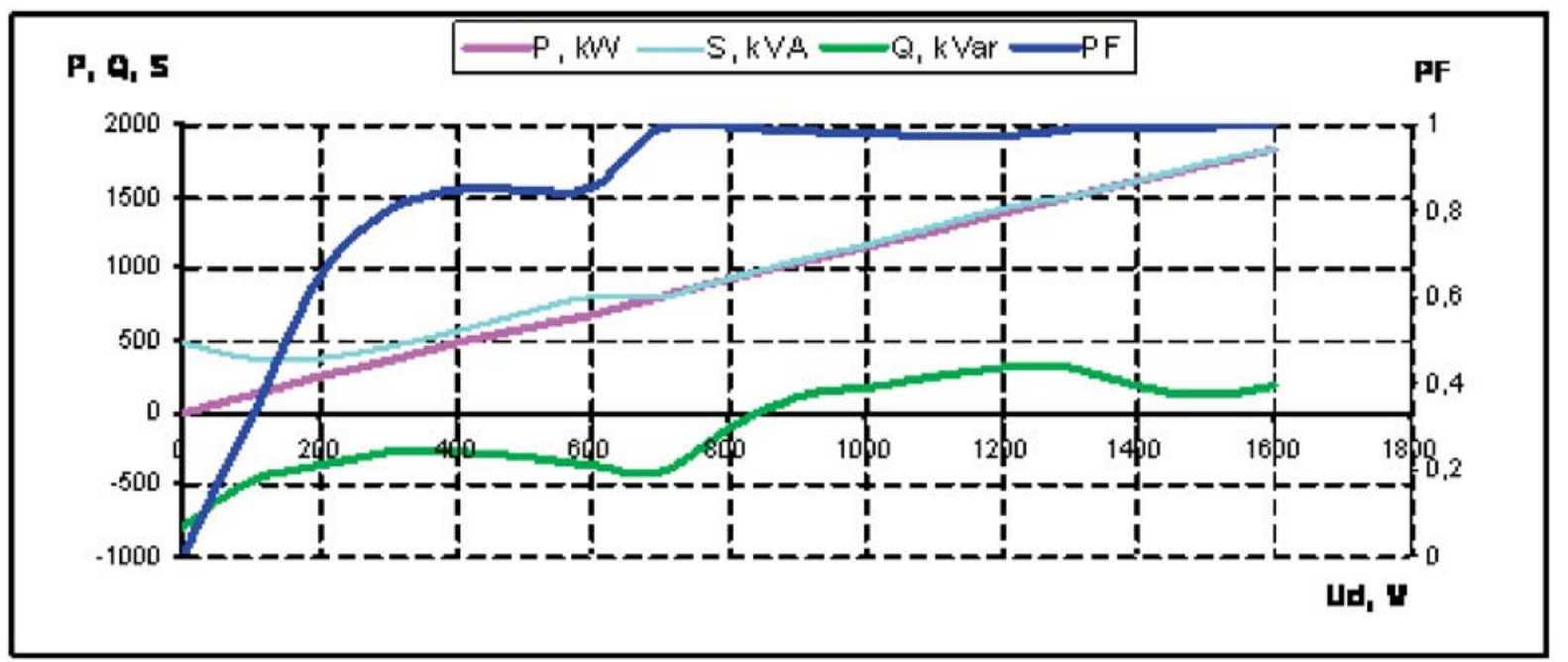

Fig. 5 Power measurement and Energy Parameters in Relation to the Average Value of the Rectified Voltage 
by incorporating these devices in the power circuitry of the EVs. There have been numerous trials of locomotives with thyristor rectifiers retrofitted with ACRP and the results from them have been described in detail in various sources. They show that $k_{M}$ for propulsion rises from 0.8 to 0.96 and the consumption of $\mathrm{Q}$ sees a significant decrease $[6,7]$.

To further study the process, a $k_{M}$ study was conducted using non-adjustable Reactive Power Compensators (RPC) set at $Q=$ $=650 \mathrm{kVAr}$ connected to a group of secondary windings in the propulsion transformer.

Figure 5 shows the power measurements and the energy parameters in propulsion mode with $1180 \mathrm{~A}$ of current supplied to the engine and the RPC turned on. They indicate that during zone-2 operation and, for the most part, during zone-1 operation, $k_{M}$ is over 0.9 .

It must be noted that decreasing the propulsion current of the EV leads to an expansion of the overcompensation area into a larger zone. As with undercompensation, overcompensation of reactive power leads to additional increase in current loads in the traction power supply system and therefore has to be avoided. Furthermore, overcompensation increases the voltage in the receiver and can cause emergency shutdown of the power supply. For these reasons onboard compensators must be of the active (adjustable) type to allow their operation to be set in accordance with the particular requirements of the $\mathrm{EV}$.

\section{Conclusion}

The trial measurements of the power consumption qualities of the upgraded electric locomotives of the 44-001 and 46-200 Series performed across numerous substations show unsatisfactory results in terms of their energy parameters. In real-life opera- tional environments, the power factor $P F_{e l}$ varies between 0.6 and 0.8 , which is substantially lower than the European norms. Figure 3 indicates that across the different patterns of movement the reactive power consumed by the locomotives is significant and that translates directly into a poor power coefficient.

Upgrading the thyristor locomotives operated within the Bulgarian railway system is unavoidable in order for the system to comply with the European norms and requirements regarding the quality of consumed power. This leaves us with two options:

A) To remove the thyristor rectifiers and retrofit four-quadrant converters with IGBT. The advantage of this option is that it will ensure optimal energy parameters across the entire adjustment range and allow recuperation. Its disadvantage is that it would not be economically justified.

B) To design and retrofit the EVs with active compensators for reactive power (ACRP). This would require a considerably smaller investment and would be more problem-free in comparison. Studies show that in terms of its energy footprint, this solution would guarantee compliance with the requirements of the European norms.

With mathematical models and trial measurements of the performance of the upgraded trains we can optimize the process of designing of active solutions to compensate the reactive power consumed by the vehicles. To improve the energy parameters and the quality of the power consumed by the vehicles, we must develop an innovative technical solution to upgrade them, namely an active compensator for reactive power (ACRP) to be connected to the secondary propulsion windings of the locomotive transformer. This, to a large extent, will eliminate the main problem related to the operation of these EVs, which is related to the consumption of considerable amounts of reactive power from the traction power supply system, and would improve the energy efficiency of the specialized railway power supply system.

\section{References}

[1] PAVLOV., G.: Increasing the Energetic Parameters of Electric Vehicles Using Input Pulse Rectifiers, Proc. of Seminar Electrical Transport Engineering (in Bulgarian), pp. 23-28, National Association of Electrical Engineers in the Transport in the Republic of Bulgaria, 2002.

[2] Dissertation thesis: Investigation of Artificial Switching Converters in Electric Rolling Stock Power-line, Technical University of Sofia, 2001

[3] DIMITROV G., PAVLOV, G.: Specialized Modular Measurement Stand for Research on Power Consumption and Energy Efficiency in Transport, International Scientific Conference Transport 2011, Sofia, Mechanics, Transport, Communications AJ, No. 3 , article No. 0597, 2011, ISSN 1312-3823.

[4] MACK GRADY, W., GILLESKIE, R. J.: Harmonics and How They Relate to Power Factor, Proc. Of the EPRI Power Quality Issues \& Opportunities Conference (PQA '93), San Diego, November 1993.

[5] MATOV P., VETSKOV, A.: Efficiency of Alternating Current Traction Network in a Uniflow Track Section, XVIII Intern. Scientific Conference Transport 2008, Sofia, Mechanics, Transport, Communications AJ, ISSN 1312-3823, No. 3, article No. $0293,2008$.

[6] DONSKOJ, D. A.: Improving Energy Performance ac Electric Locomotives. Proc. of Scientific and Technical Conference ScienceTransport-2006, Moscow Engineering, 2006

[7] DONSKOJ, D. A.: Improving the Efficiency of Electric Traction on AC. J. of Electronics and Electrical Equipment in the Transport. No. 5,2006 